\title{
Hepatitis C Viral Kinetics in Special Populations
}

\author{
Harel Dahari, $\mathrm{PhD}^{1}{ }^{1}{ }^{*}$, Jennifer E. Layden-Almer, $\mathrm{MD} \& \mathrm{PhD}^{1}$, Alan S. Perelson, $\mathrm{PhD}^{2}$, and \\ Thomas J. Layden, MD ${ }^{1}$ \\ 1Department of Medicine, Section of Hepatology, The University of Illinois at Chicago 840 S. Wood Street \\ MC787, Chicago, IL 60612 \\ 2Theoretical Biology and Biophysics, MS-K710, T-10, Los Alamos National Laboratory, Los Alamos, NM \\ 87545
}

\begin{abstract}
Mathematical models of hepatitis $\mathrm{C}$ viral (HCV) kinetics provide a means of estimating the antiviral effectiveness of therapy, the rate of virion clearance and the rate of loss of HCV-infected cells. They have also proved useful in evaluating the extrahepatic contribution to HCV plasma viremia and they have suggested mechanisms of action for both interferon- $\alpha$ and ribavirin. Viral kinetic models can explain the observed HCV RNA profiles under treatment, e.g., flat partial response, biphasic and triphasic viral decay and viral rebound. Current therapy with (pegylated) interferon- $\alpha$ and ribavirin has a poorer success in patients having insulin resistance, hepatic fibrosis, African American ethnicity, HCV/HIV-coinfection, HCV genotype-1 and high baseline viral load. The use of mathematical modeling and statistical analysis of experimental data have been useful in understanding some of these treatment obstacles.
\end{abstract}

\section{Introduction}

Approximately $3 \%$ of persons worldwide are infected with hepatitis C virus (HCV) [1]. Current therapy consisting of pegylated interferon- $\alpha$ (PEG-IFN) and ribavirin (RBV) produces sustained virological response (SVR) rates of approximately $50 \%$ overall, with no effective alternative treatment for non-responders. Host factors including obesity, insulin resistance, hepatic fibrosis, and African American ethnicity as well as viral factors such as genotype 1 and high baseline viral load are associated with lower SVR rates. Mathematical modeling serves as an important tool in understanding why these host and viral factors lower SVR rates.

\section{HCV RNA kinetics patterns}

\section{HCV RNA kinetics before and during therapy}

During chronic HCV infection, the level of serum HCV RNA does not vary significantly $(<0.5$ $\log$ ) on time scales of weeks to months [2]. However, when patients chronically infected with $\mathrm{HCV}$ are treated with interferon- $\alpha$ (IFN) or IFN plus ribavirin, HCV RNA generally declines after a $7-10$ hour delay. The typical decline is biphasic and consists of a rapid first phase lasting for approximately $1-2$ days during which HCV RNA, on average, may fall 1 to $2 \operatorname{logs}$ in genotype 1 infected patients [3] and as much as 3 to 4 logs in genotype 2 infected patients [4]. Subsequently, a slower second phase of HCV RNA decline ensues. Triphasic viral declines also have been observed in some patients [5-7]. A triphasic decline consists of a first phase (1

*Corresponding author: Dahari H (E-mail: daharih@uic.edu), Tel: (312) - 413-2851, Fax: (312) -413-5604, Layden-Almer JE (E-mail: jlayde1@uic.edu) and Layden TJ (E-mail: tjlayden@uic.edu), Tel: 312-996-6651, Fax: 312-996-5103, Perelson AS (E-mail: asp@lanl.gov), Tel:505-667-6829, Fax: 505-665-3493. 
- 2 days) with rapid virus load decline followed by a shoulder phase (4-28 days) - in which virus load decays slowly or remains constant - and a third phase of renewed viral decay. In nonresponders, there may be no viral decline (null response) or a first phase followed no second-phase decline (flat partial response) or rebound to baseline level. In Figure 1, we show some typical examples of these viral load profiles under therapy.

\section{HCV RNA kinetics during and post liver transplantation}

Detailed studies of HCV RNA kinetics during the anhepatic phase and immediately after liver transplantation were recently reported [8-11]. Most patients showed a rapid viral decline during the anhepatic phase followed by a similar decline several hours after graft reperfusion. Thereafter, some patients reached undetectable viral levels and then had a viral rebound, or had a viral rebound immediately following the rapid decline. Others had a biphasic decline before the viral rebound, with either a viral plateau or a slower decline. After approximately 4 to 55 days post-transplantation, viral concentrations exceed pre-transplantation levels.

\section{Models of HCV RNA kinetics}

A new field called viral dynamics emerged in 1995 with an initial goal of modeling human immunodeficiency virus (HIV) infection and treatment $[12,13]$. This approach was adapted in 1998 by Neumann et al.[3] to study the kinetics of the HCV RNA response to high dose daily IFN. Since then much progress has been made in our understanding of HCV RNA dynamics during primary infection [14], under treatment and during (and post) liver transplantation [9, 10]. Kinetic models have taken into consideration the different pharmacokinetics of standard and pegylated forms of interferon [15,16], and have incorporated alanine aminotransferase kinetics $[17,18]$ and aspects of host responses to provide a more comprehensive picture of the biology underlying changes in HCV RNA levels during therapy. Models and their use in clinical practice are explained below and summarized in Table 1 and Table 2, respectively.

\section{Model of HCV RNA infection and treatment}

A model of HCV infection and treatment introduced by Neumann et al. [3] has proven extremely useful in analyzing HCV RNA decay under drug therapy.

$$
\begin{aligned}
& \frac{d T}{d t}=s-d_{T} T-(1-\eta) \beta V T \\
& \frac{d I}{d t}=(1-\eta) \beta V T-\delta I \\
& \frac{d V}{d t}=(1-\varepsilon) p I-c V
\end{aligned}
$$

Cells that are susceptible to HCV infection are termed target cells, $T$. The model assumes that target cells are produced at a constant rate $\mathrm{s}$, and die at rate $d_{T}$ per cell. Upon interaction with $\mathrm{HCV}$ these cells become productively infected cells, $I$, with infection rate constant $\beta$. Infected cells produce viral particles (virions), $V$, at rate $p$ per infected cell and are lost at a rate $\delta$ per infected cell. Virions are cleared at a constant rate $c$. Therapy is assumed to act by partially blocking virion production, with effectiveness $\varepsilon$, and by reducing the rate of infection, with effectiveness $\eta$, where $\varepsilon$ and $\eta$ vary between 0 and 1, with 1 meaning $100 \%$ effectiveness.

\section{First-phase HCV RNA decline during interferon- $\alpha$ based therapy}

Our initial studies showed that there was a dose-dependent reduction in HCV RNA levels over the first 24-48 hours followed by a slower second phase decline in patients that were given different doses of IFN- $\alpha 2 b[3,19]$. To explain this, Neumann et al. [3] predicted that IFN must interfere with the ability of infected cells to produce or release virus, and this has recently been confirmed in various cell-culture systems [20]. In addition, Neumann et al. [3] suggested that if one examines only the first phase and assumes that on this time scale the target and infected 
cell number remains close to their level before therapy, then the model (1) predicts that viral load will decline from its baseline value, $V_{0}$, according to the equation:

$$
V(t)=V_{0}\left[1-\varepsilon+\varepsilon e^{-c\left(t-t_{0}\right)}\right], \quad t>t_{0}
$$

Viral decay occurs after time $t_{0}$, which corresponds to the pharmacologic delay (7- $10 \mathrm{hr}$ ) [3]. Neumann et al. fitted this equation to the experimental data collected over the first 2 days of treatment in each patient and the parameters $c$ and $\varepsilon$ were estimated. The mean intrinsic clearance rate was $c=6.2 \pm 1.8$ day $^{-1}$, giving a viral half life (i.e., the time it takes for half the virus to be cleared from circulation) of 2.7 hours (range, 1.5 to $4.6 \mathrm{hr}$ ). IFN effectiveness demonstrated strong dose dependency; with an average drug effectiveness of $\varepsilon=81 \%$ under 5 million IU and $\varepsilon \sim 95 \%$ under 10 and 15 million IU IFN doses. Equation (2) predicts that the viral load will decline to the value $(1-\varepsilon) V_{0}$ at times longer than $1 / c$, the average free virion lifetime in serum. Thus, the effectiveness in blocking virion production or release is approximately given by $\varepsilon=1-\mathrm{V}_{1 \mathrm{st}} / \mathrm{V}_{0}$, where $\mathrm{V}_{1 \mathrm{st}}$ represents viral load at the end of the first phase.

The concepts of drug effectiveness in blocking $\mathrm{HCV}$ production and first and second phase decay have led to the following observations:

- RBV does not affect the first phase decay of HCV RNA when given in combination with (PEG)-IFN [6,21].

- African American patients exhibit significantly lower drug effectiveness (88.6\% vs. 98.2\%) than Caucasians [22].

- Patients with high levels of insulin resistance have significantly lower viral load drops from baseline than patients with low insulin resistance [23].

- Pharmacokinetics studies have shown obese patients to have lower maximum serum IFN concentrations after a dose of IFN and longer times to maximal serum concentration compared to non-obese patients [24]. Based on re-analysis of published data, obese patients had lower IFN effectiveness compared to non-obese patients (unpublished data).

- The presence of steatosis impairs the early reduction of viral load during treatment in patients infected with HCV [25].

- Larger viral load drops were observed in HCV genotype 3 cirrhotic patients (mean $\varepsilon=99 \%$ ) than in HCV genotype 1 cirrhotic patients (mean $\varepsilon=80 \%$ ) [26 $]$.

- Drug effectiveness is higher in HCV genotype 2 patients (mean $\varepsilon=99.7 \%$ ) than in HCV genotype 1 patients (mean $\varepsilon=95.3 \%$ ) [4].

- Nonresponders have lower drug effectiveness than naïve patients [27].

- Interestingly, there was no significant difference in first phase decline between children (mean $1.2 \pm 0.8 \log \mathrm{IU} / \mathrm{ml})$ and adults $(0.9 \pm 0.6 \log \mathrm{IU} / \mathrm{ml})[28]$.

- HCV genotype 1 virion half-life was estimated to be longer in HIV-coinfected patients (mean $7 \mathrm{hr}$ ) [29] than in genotype $1 \mathrm{HCV}$ monoinfected patients (mean $2.7 \mathrm{hr}$ ) [3].

- HCV half-life was significantly shorter in genotype $2 \mathrm{HCV}$ (mean $1.8 \mathrm{hr}$ ) than in genotype 1 patients [4].

Importantly, attempts to predict sustained virological response (SVR; i.e., undetectable HCV RNA level 6 months after stopping treatment) from first-phase decline were also studied. Layden et al. [30] as well as Jessner et al. [31], showed that treatment non-response could be 
predicted after twenty-four hours with very high predictive value for non-response. In a retrospective study, Layden et al. [30] showed that in patients who did not have an adequate IFN effectiveness and low enough viral load at day one of therapy, $100 \%$ went on to be nonresponders. Jessner et al. [31] showed that in patients who did not have at least a one log drop in viral load at day one of therapy, $100 \%$ were non-responders. While the standard of care has changed since these initial reports, making it hard to utilize the exact criteria found in these studies, it is interesting to note that numerous subsequent studies have shown results allowing for increasingly early prediction of treatment non-response, e.g, [32]. In a recent study, Sherman et al. [33] found that in HCV/HIV-coinfected patients treated with PEG-IFN+RBV, IFN+RBV effectiveness $\geq 90 \%$ was associated $(p=0.02)$ with viral clearance. Although these studies are small, they serve as important sign posts for larger ones.

Lastly, in the quasi-steady state in which viral load is constant before therapy, the rate of viral production has to equal the rate of virion clearance (i.e., $\mathrm{cV}_{0}$ ). Since the viral clearance rate, $c$, and baseline viral load, $V_{0}$, are known, one can estimate the total amount of virus cleared and, hence produced per day. This calculation shows that about $10^{11}-10^{12}$ virions are produced per day [3]. Since HCV mutates when it replicates about as fast as HIV, drug resistance presents a challenge for treatments under development using small molecule inhibitors, e.g., HCV protease inhibitors [34].

\section{Second-phase HCV RNA decline under therapy}

The assumption of a constant number of infected cells was relaxed by Neumann et al. during the prolonged second phase decline [3]. Solving equation (1), but assuming that target cells (T) are still at steady state during the first 14 days of therapy, model (1) predicts

$$
V(t)=V_{0}\left(A e^{-\lambda_{1}\left(t-t_{0}\right)}+(1-A) e^{-\lambda_{2}\left(t-t_{0}\right)}\right)
$$

where

$$
\lambda_{1,2}=\frac{1}{2}\left(c+\delta \pm \sqrt{(c-\delta)^{2}+4(1-\varepsilon)(1-\eta) c \delta}\right) \quad \text { and } \quad A=\frac{\varepsilon c-\lambda_{2}}{\lambda_{1}-\lambda_{2}} .
$$

Equation (3) predicts that the viral load under treatment will always decrease to the uninfected steady state following an initial rapid viral decline (first phase) followed by a slower decay (second phase), with rates $\lambda_{1}$ and $\lambda_{2}$, respectively (Fig. 1). Indeed, the typical HCV RNA decay during therapy with (PEG) IFN alone or in combination with RBV is biphasic and can be fitted to Eq. (3) $[3,6,21,35]$. These fits are usually done assuming $\eta=0$, and yield an estimate of the death rate of infected cells, $\delta$. The assumption $\eta=0$ is made because it can be shown that for large values of $\varepsilon$, as are obtained by many therapies, the effect of $\eta$ on the viral load decline predicted by Eq. (3) is negligible [3]. The value of $\eta$ can also be fixed to non-zero values, but in general, the available data are insufficient to estimate it. Of note, Eq. (3) predicts only a biphasic decline and thus, in some patients, $\delta$ could not be estimated because the second-phase viral decline is flat or viral rebound occurred.

There is no direct evidence that $\delta$ estimated from the second phase of HCV RNA decline is the loss rate of infected cells. Interestingly, Neumann et al. [3] and Zeuzem et al. [36] noted that $\delta$ was correlated with baseline alanine aminotransferase (ALT), a surrogate marker of liver cells necrosis, so that the higher the initial ALT level the larger the value of $\delta$ under therapy with IFN or PEG-IFN. In addition, an inverse correlation was found between baseline HCV RNA and $\delta$ [3]. Recently, Pilli et al. [37••] found that patients with rapid viral declines had better HCV-specific CD8 cell proliferative responses at baseline. These observations support the concept that $\delta$ represents the loss rate of infected cells predominately due to cellular immune responses. However, a biphasic decay of HCV RNA was recently observed in severe combined 
immunodeficient chimeric mice with human hepatocytes during treatment with PEG-IFN [38]. The second phase slope is interesting as it still occurs in immunodeficient mice [38]. Since a biphasic decay pattern was also observed in IFN treated Huh7 cells harboring HCV replicons, it may be possible that the second phase decay observed in immunodeficient mice, and in treated patients, reflects not only the death/loss of productively-infected cells but also the HCV RNA kinetics within cells [39].

Overall, Eq. (3) represents a fundamental breakthrough in the field of HCV treatment. The value of $\delta$, estimated over the first two of weeks of therapy as described above, was predictive of viral negativity at 12 weeks of therapy and no patient that exhibited a $\delta<0.1$ day $^{-1}$ achieved viral negativity ( $<100$ copies HCV-RNA/ml) 12 weeks into treatment [3]. In a study comparing viral kinetics between African Americans and Caucasians, African Americans displayed a significantly decreased mean $\delta$ compared to Caucasians [22]. However, from Eq. (3) the slower exponential decay rate, $\lambda_{2}$ approximates $\delta$ only when the total drug efficacy is close to 1 . Thus, for efficacies less than 1 there is continuing infection of new cells and hence a slower secondphase decline occurs, and the second-phase slope is only a minimal estimate of $\delta$. Indeed, Layden-Almer et al. [22] noted that when controlling for IFN effectiveness, $\delta$ was similar between African Americans and Caucasians. Since a low drug efficacy could affect the estimate of $\delta$, one can simply estimate the second phase slope of HCV RNA decay in order to predict the outcome of treatment (i.e., SVR). Studies of HCV kinetics showed that a second-phase slope $<0.3 \mathrm{log} /$ week is predictive of nonresponse [26•,40]. In particular, Medeiro-Filho et al. [26•] found that the HCV RNA second-phase decay slope in genotype 3 cirrhotic patients was significantly faster than in genotype 1 cirrhotic patients. This could be explained by a less potent immune response or drug effectiveness [41] in genotype 1 cirrhotic patients than in genotype 3 cirrhotic patients. Conversely, Neumann et al. [28] indicated that $62 \%$ of treated children had a second phase slope $<0.3 \log /$ week but 4 out of these 8 children nevertheless achieved SVR. However, larger studies are needed to determine the viral kinetic predictors of SVR in children.

\section{Modeling of interferon- $\alpha$ pharmacokinetics and pharmacodynamics}

We noted above that Eq. (3) does not predict viral rebound following the first-phase viral decay. When PEG-IFN $\alpha 2 b$ is used, there is a reasonable concern that some of the observed viral rebounds are due to changes in drug efficacy between weekly dosing (Fig. 1, dashed line). Indeed, viral increase tends to occur when serum levels of PEG-IFN are low towards the end of the 7-day dosing interval $[15,16,42]$. Thus, one could allow $\varepsilon$ to depend on the PEG-IFN serum concentration, $C$, according to the pharmacodynamic model

$$
\varepsilon(t)=\frac{C^{n}(t-\tau)}{E C_{50}^{n}+C^{n}(t-\tau)}
$$

where the constant $E C_{50}$ is the PEG-IFN concentration at which the drug's effectiveness in blocking viral production is half its maximum, $n$ is a parameter called the Hill coefficient, and the time delay $\tau$ takes into account that IFN must bind cellular receptors and initiate a signaling cascade before a drug affect is observed.

To model both HCV RNA and PEG-IFN concentrations in $21 \mathrm{HCV} / \mathrm{HIV}$-coinfected patients during the first 2 weekly PEG-IFN doses, Talal et al. [16••] replaced $\varepsilon$ by $\varepsilon(\mathrm{t})$, given by equation (4), and then numerically solved Eq. (1) with $T$ held constant. They showed that viral rebound, observed in some patients, occurred when drug concentration was low relative to $\mathrm{EC}_{50}$. Interestingly, the authors found that although drug concentration per se was not associated with SVR, some features such as the maximum drug effectiveness, computed from Eq. (4), and the weekly average drug concentration divided by EC50 were correlated with SVR [16]. 
Clearly, models with time-varying drug effects are more accurate than models assuming constant efficacy. Such models require drug concentrations that are not commonly measured in clinical studies. New models that allow the effectiveness to vary but which are independent of pharmacokinetic data, such as that of Bekkering et al. [43], are needed and are under development by Perelson and colleagues.

\section{Critical drug efficacy}

We noted above that equation (3) only predicts biphasic viral drops if $\delta$ is very small or zero, corresponding to a flat partial response and an infected cell half-life of months to years (Fig. 1). Such small values of $\delta$ are not biologically realistic since they predict that infected cells live longer than unininfected ones. Recently, using equation (1) with target cell levels allowed to vary with time, the notion of a critical drug efficacy, $\varepsilon_{c}$, for the treatment of HCV [44] was introduced, analogous to previous efforts in the field of HIV treatment $[45,46]$. If a drug blocks both infection and viral production, then the total efficacy, defined as $\varepsilon_{t o t}=1-(1-\varepsilon)(1-\eta)$, must be greater than $\varepsilon_{c}$ for continuous viral decay. If $\varepsilon_{t o t}<\varepsilon_{c}$, then the theory predicts [44] that HCV RNA levels initially decline but ultimately stabilize at a new steady state despite continued therapy and high $\delta$ i.e., a flat-partial response occurs (Fig. 1). The critical efficacy is

$$
\varepsilon_{c}=1-\frac{d_{T} c \delta}{s p \beta} .
$$

The critical condition $\varepsilon_{\text {tot }}>\varepsilon_{c}$ is equivalent to the standard condition from epidemiology that the basic reproduction number $R_{0}<1$ for an epidemic to die out, where $R_{0}=\frac{s p \beta}{d_{T} c \delta}$. In addition, the critical efficacy is approximately equal to the fraction of $\mathrm{HCV}$-infected hepatocytes at baseline [44]. Thus, theoretically, patients with a high fraction of HCV-infected hepatocytes will have high $\varepsilon_{c}$ and hence will have a poorer chance of achieving SVR. Interestingly, Pal et al. [47] showed that advanced liver disease is associated with a higher fraction of HCV-infected hepatocytes and lower SVR rates $(<29 \%)$ than for patients without liver disease $[25,26,48$, 49].

In summary, the notion of critical drug effectiveness may explain why some patients do not achieve SVR. In particular, a flat partial response, i.e., a rapid first phase of viral decay followed by a flat second phase can be the consequence of drug effectiveness being too low.

\section{Triphasic HCV RNA decay}

In some patients, regardless of drug regimen (i.e., IFN, PEG-IFN, IFN+RBV, PEG-IFN [5,6, 43] and PEG-IFN + telaprevir [7]), a triphasic HCV RNA decline was observed (Fig. 1). It was suggested by Hermann et al. [6] that the final phase of a triphasic viral decline could be due to a delayed enhancement of an anti-HCV immune response by IFN or PEG-IFN. Moreover, Hermann et al. suggested that this immune enhancement is greater when RBV is added to IFN/ PEG-IFN as the final phase slope was found to be larger when RBV was present. While a delayed anti-HCV immune response might be triggered by IFN and RBV, the shoulder phase is difficult to explain in the Hermann et al. [6] and Neumann et al. [3] models as it requires the assumption that the infected cell loss rate, $\delta$ be close to zero in order to generate a flat phase. To address this difficulty, Dahari et al. [44,50] proposed an extension of model (1) that includes density-dependent proliferation terms for both uninfected and infected hepatocytes. The extended model is given by 


$$
\begin{aligned}
& \frac{d T}{d t}=S+r_{T} T\left(1-\frac{T+I}{T_{\max }}\right)-d_{T} T-(1-\eta) \beta V T \\
& \frac{d I}{d t}=(1-\eta) \beta V T+r_{I} I\left(1-\frac{T+I}{T_{\max }}\right)-\delta I \\
& \frac{d V}{d t}=(1-\varepsilon) p I-c V
\end{aligned}
$$

Uninfected and infected hepatocytes can proliferate with maximum proliferation rates $r_{T}$ and $r_{I}$, respectively, according to a blind homeostasis process in which there is no distinction between infected and uninfected cells [14]. Due to the burdens of supporting HCV replication, Dahari and colleagues assume that infected cells may proliferate slower than uninfected cells, i.e., $r_{I} \leq r_{T}$. If the total hepatocyte population, $T+I$, reaches a maximum level, $T_{\max }$, hepatocyte proliferation stops. The corresponding critical drug efficacy, $\varepsilon_{c}$, for model (6) is [50]

$$
\varepsilon_{c}=1-\frac{c\left(\delta T_{\max }+r_{I}\left(\bar{T}_{0}-T_{\max }\right)\right)}{p \beta T_{\max } \bar{T}_{0}} .
$$

The extended model predicts that the HCV RNA decline may be biphasic or triphasic when $\varepsilon_{\text {tot }}>\varepsilon_{c}[44,50]$. The authors predicted that a shoulder phase, and hence a triphasic viral decay, only occurs if the number of uninfected hepatocytes is much lower than the number of infected hepatocytes before therapy and uninfected hepatocytes proliferate faster than infected cells. Under these conditions, the generation of new uninfected hepatocytes to a level at which they slow infected cell proliferation takes a substantial length of time. This delay causes the shoulder phase. Thus, the shoulder phase in triphasic responses (Fig. 1), may be a simple consequence of liver homeostasis in which for some period of time the proliferation of infected hepatocytes compensates for their loss/death. In such circumstances the slope of the shoulder phase does not represent $\delta$.

Using equation (6) one can predict triphasic viral decay during (PEG)-IFN treatment without invoking a delayed effect of interferon- $\alpha$ in enhancing the anti-HCV immune response.

Furthermore, by including hepatocyte proliferation in the Dixit et al. [21] model of RBV action, Dahari et al. [50॰] predicted triphasic viral decay and enhancement of third phase decay by $\mathrm{RBV}$ without assuming that RBV increases immune responses causing an increase in the infected cell death rate.

Note that although equation (6) gives new insights into HCV kinetics, it introduces additional parameters, such as infected and uninfected hepatocyte proliferation rates, that may not be possible to uniquely estimate from HCV RNA data alone. In addition, measurements of the baseline fraction of $\mathrm{HCV}$-infected hepatocytes are needed to support model predictions. Thus, this model, while of great theoretical utility, may not be a practical alternative to equation (3) of Neumann et al. [3] until methods become available to obtain quantitative information about hepatocyte proliferation in $\mathrm{HCV}$-infected patients.

\section{Modeling HCV kinetics during and after liver transplantation}

Liver transplantation (LT) in end-stage HCV-related liver disease is currently the only lifesaving alternative. However, infection of the liver graft after transplantation is almost universal and leads to an accelerated rate of chronic hepatitis and cirrhosis. This inevitable post-transplant infection may be related to the existence of an auxiliary HCV compartment. Thus, the putative existence of a second replication compartment is an important issue in optimizing the treatment of $\mathrm{HCV}$ infection and avoiding the post LT recurrence of HCV infection.

Theoretically, the existence of an extraheptic replication compartment could be ruled out if the rapid viral decline during the anhepatic phase is a single exponential. Thus, if we assume that removal of the liver results in a complete lack of productively infected cells (that is $I=T=0$ ) during the the anhepatic phase, then equation (1) reduces to 


$$
\frac{d V}{d t}=-c V
$$

and HCV RNA is predicted to decay due to viral clearance in the absence of production. Dahari et al. [9] and Powers et al. [10] used equation (8) to analyze viral decline during the anhepatic phase and after graft reperfusion but before virion production from the new graft starts. In 12 patients (44\%) from Dahari et al. and 3 patients (50\%) from Powers et al. a single exponential decline was seen before viral increase. Thus, these patients have either a second compartment with a relatively low contribution to viral production or no second compartment. The remaining patients ( $\sim 53 \%$ in both studies) had a biphasic viral decay or a viral plateau, before the viral increase, suggesting the existence of a second replication compartment.

To estimate the extrahepatic HCV contribution to plasma virus, Dahari et al. [9] developed a model with a putative extrahepatic compartment, allowing diffusion and equilibration of virus between the two compartments. This model was fitted to the data and estimated that any extrahepatic compartments would be responsible for approximately $3 \%$ of virus in circulation. Powers et al. [10] also predicted the same extrahepatic contribution after including in equation (8) the dilution effects of fluid input during surgery.

To model the post anhepatic resurgence in viral concentration, Powers et al. [10] assumed that because viral resurgence is rapid the number of target cells during this perioid was mainly affected by viral infection at rate $\beta \mathrm{VT}$, rather than by new hepatocyte production or death. In this scenario, model (1) reduces to

$$
\begin{aligned}
& \frac{d T}{d t}=-\beta V T \\
& \frac{d I}{d t}=\beta V T-\delta I \\
& \frac{d V}{d t}=p I-c V
\end{aligned}
$$

By fitting model (9) to serum HCV RNA concentrations during resurgence, $\delta$ was estimated to be close to 0 , possibly reflecting the effects of immunosuppression on cell-mediated immune responses. In addition, the authors estimated that viral resurgence begins when much less than $1 \%$ of the engrafted liver's hepatocytes are infected and suggested that antiviral therapy should begin soon after, or before LT. Moreover, they estimated that on average 19\% (range $0.2 \%$ to $90 \%$ ) of hepatocytes are productively infected at the steady state obtained after postoperative resurgence. It should be noted that the data used by Powers et al. was obtained from patients obtaining grafts from deceased donors (DDs).

An interesting viral kinetics study by Schiano et al. [11 • $]$ compared the fall and rise of HCV RNA in recipients of grafts from living donors (LDs; $N=11)$ and DDs $(\mathrm{N}=15)$. The authors noted that during the first 7 hours after graft reperfusion there was a greater (trend, $\mathrm{p}=0.16$ ) HCV RNA decrease in LD recipients than in DD recipients. Moreover, HCV RNA levels increased significantly more rapidly $(\mathrm{p}<0.01)$ in LD recipients than in DD recipients between days 1 and 3 post LT. The results from this study suggest that HCV interacts differently with LD grafts than with DD grafts. Since the population of dividing cells in LD grafts is expected to be higher than in DD grafts, and higher ALT level persisted throughout the study in LD patients, model (9) would need to be modified to properly analyze HCV kinetics in LD graft recipients.

\section{Conclusion}

Viral kinetic modeling has stimulated the collection of frequent HCV RNA data from which various kinetic patterns, such as rapid virological responses, have been identified. Using the response over the first few days of therapy has allowed estimates of drug effectiveness to be 
made, and response over longer periods have lead to predictions of whether a patient will attain SVR. Thus, we are moving toward the point where viral kinetics may allow clinicians to better select, optimize and target therapy for those patients with expected poor responses.

\section{Acknowledgements}

Portions of this work were done under the auspices of the U. S. Department of Energy under contract DEAC52-06NA25396 and supported by NIH grants AI28433, AI065256, RR06555, and P20-RR18754 (ASP). H.D is supported by the University of Illinois Gastrointestinal and Liver Disease (GILD) Association.

\section{References and Recommended Reading}

Papers of particular interest, published recently, have been highlighted as:

- Of importance

•• Of major importance

1. NIH. National Institutes of Health Consensus Development Conference: management of hepatitis C: 2002. Hepatology 2002;36:S3-20. [PubMed: 12407572]

2. Nguyen TT, Sedghi-Vaziri A, Wilkes LB, et al. Fluctuations in viral load (HCV RNA) are relatively insignificant in untreated patients with chronic HCV infection. J Viral Hepat 1996;3:75-8. [PubMed: 8811641]

3. Neumann AU, Lam NP, Dahari H, et al. Hepatitis C viral dynamics in vivo and the antiviral efficacy of interferon-alpha therapy. Science 1998;282:103-7. [PubMed: 9756471]

4. Neumann AU, Lam NP, Dahari H, et al. Differences in viral dynamics between genotypes 1 and 2 of hepatitis C virus. J Infect Dis 2000;182:28-35. [PubMed: 10882578]

5. Bergmann CC, Layden JE, Levy-Drummer RS, et al. Clinical implications of a new tri-phasic model for hepatitis C viral kinetics during IFN-alpha therapy. Hepatology 2001;34:345A.

6. Herrmann E, Lee JH, Marinos G, et al. Effect of ribavirin on hepatitis C viral kinetics in patients treated with pegylated interferon. Hepatology 2003;37:1351-8. [PubMed: 12774014]

7. Kieffer TL, Sarrazin C, Miller JS, et al. Telaprevir and pegylated interferon-alpha-2a inhibit wild-type and resistant genotype 1 hepatitis $C$ virus replication in patients. Hepatology 2007;46:631-9. [PubMed: 17680654]

8. Garcia-Retortillo M, Forns X, Feliu A, et al. Hepatitis C virus kinetics during and immediately after liver transplantation. Hepatology 2002;35:680-687. [PubMed: 11870384]

9. Dahari H, Feliu A, Garcia-Retortillo M, Forns X, Neumann AU. Second hepatitis C replication compartment indicated by viral dynamics during liver transplantation. J Hepatol 2005;42:491-8. [PubMed: 15763335]

10. Powers KA, Ribeiro RM, Patel K, Pianko S, et al. Kinetics of hepatitis C virus reinfection after liver transplantation. Liver Transpl 2006;12:207-216. [PubMed: 16447184]

11••. Schiano TD, Gutierrez JA, Walewski JL, et al. Accelerated hepatitis C virus kinetics but similar survival rates in recipients of liver grafts from living versus deceased donors. Hepatology 2005;42:1420-8. [PubMed: 16317672] This study is important because little information is available regarding early HCV RNA kinetics in living donors graft recipients.

12. Ho DD, Neumann AU, Perelson AS, et al. Rapid turnover of plasma virions and CD4 lymphocytes in HIV-1 infection. Nature 1995;373:123-6. [PubMed: 7816094]

13. Wei X, Ghosh SK, Taylor ME, et al. Viral dynamics in human immunodeficiency virus type 1 infection. Nature 1995;373:117-22. [PubMed: 7529365]

14. Dahari H, Major M, Zhang X, Mihalik K, et al. Mathematical modeling of primary hepatitis C infection: Noncytolytic clearance and early blockage of virion production. Gastroenterology 2005;128:1056-66. [PubMed: 15825086]

15. Powers KA, Dixit NM, Ribeiro RM, et al. Modeling viral and drug kinetics: hepatitis C virus treatment with pegylated interferon alfa-2b. Semin Liver Dis 2003;23(Suppl 1):13-8. [PubMed: 12934163] 
16••. Talal AH, Ribeiro RM, Powers KA, et al. Pharmacodynamics of PEG-IFN alpha differentiate HIV/ HCV coinfected sustained virological responders from nonresponders. Hepatology 2006;43:94353. [PubMed: 16761329]This is the first modeling paper that couples PEG-IFN- $\alpha-2 b$ plasma concentrations and effectiveness in blocking HCV production.

17. Ribeiro RM, Layden-Almer J, Powers KA, et al. Dynamics of alanine aminotransferase during hepatitis C virus treatment. Hepatology 2003;38:509-517. [PubMed: 12883496]

18. Colombatto P, Civitano L, Oliveri F, et al. Sustained response to interferon-ribavirin combination therapy predicted by a model of hepatitis $\mathrm{C}$ virus dynamics using both HCV RNA and alanine aminotransferase. Antivir Ther 2003;8:519-30. [PubMed: 14760885]

19. Lam NP, Neumann AU, Gretch DR, et al. Dose-dependent acute clearance of hepatitis C genotype 1 virus with interferon alfa. Hepatology 1997;26:226-231. [PubMed: 9214474]

20. Blight KJ, Kolykhalov AA, Rice CM. Efficient initiation of HCV RNA replication in cell culture. Science 2000;290:1972-1974. [PubMed: 11110665]

21. Dixit NM, Layden-Almer JE, Layden TJ, Perelson AS. Modelling how ribavirin improves interferon response rates in hepatitis C virus infection. Nature 2004;432:922-4. [PubMed: 15602565]

22. Layden-Almer JE, Ribeiro RM, Wiley T, et al. Viral dynamics and response differences in HCVinfected African American and white patients treated with IFN and ribavirin. Hepatology 2003;37:1343-50. [PubMed: 12774013]

23. Bortoletto G, Realdon S, Dal Pero F, et al. Insulin resistance (IR) defined by the homeostasis model of assessment insulin resistance (HOMA-IR) index has a direct effect on early viral kinetics during pegylayted-interferon therapy for chronic hepatitis C. Hepatology 2007;46:361A.

24. Lam NP, Pitrak D, Speralakis R, et al. Effect of obesity on pharmacokinetics and biologic effect of interferon-alpha in hepatitis C. Dig Dis Sci 1997;42:178-85. [PubMed: 9009135]

25. Westin J, Lagging M, Dhillon AP, et al. Impact of hepatic steatosis on viral kinetics and treatment outcome during antiviral treatment of chronic HCV infection. J Viral Hepat 2007;14:29-35.

[PubMed: 17212641]

26•. Medeiros-Filho JE, de Carvalho Mello IM, Pinho JR, et al. Differences in viral kinetics between genotypes 1 and 3 of hepatitis $C$ virus and between cirrhotic and non-cirrhotic patients during antiviral therapy. World J Gastroenterol 2006;12:7271-7. [PubMed: 17143940]This study shows the effect of liver histology on viral kinetics in patients infected with HCV. Possible other interpretations of the results of this study are discussed in reference 41.

27. Cotler SJ, Layden JE, Neumann AU, Jensen DM. First phase hepatitis c viral kinetics in previous nonresponders patients. J Viral Hepat 2003;10:43-9. [PubMed: 12558911]

28. Neumann AU, Mohan P, Lutchman G, et al. Hepatitis C viral linetics in children treated with PEGIFN alfa-2a. Hepatology 2004;40

29. Torriani FJ, Ribeiro RM, Gilbert TL, et al. Hepatitis C virus (HCV) and human immunodeficiency virus (HIV) dynamics during HCV treatment in HCV/HIV coinfection. J Infect Dis 2003;188:1498507. [PubMed: 14624375]

30. Layden JE, Layden TJ, Reddy KR, et al. First phase viral kinetic parameters as predictors of treatment response and their influence on the second phase viral decline. J Viral Hepat 2002;9:340-5. [PubMed: 12225328]

31. Jessner W, Gschwantler M, Steindl-Munda P, et al. Primary interferon resistance and treatment response in chronic hepatitis C infection: a pilot study. Lancet 2001;358:1241-2. [PubMed: 11675067]

32. Dahari H, Markatou M, Zeremski M, et al. Early ribavirin pharmacokinetics, HCV RNA and alanine aminotransferase kinetics in HIV/HCV co-infected patients during treatment with pegylated interferon and ribavirin. J Hepatol 2007;47:23-30. [PubMed: 17412448]

33. Sherman KE, Shire NJ, Rouster SD, et al. Viral kinetics in hepatitis $\mathrm{C}$ or hepatitis C/human immunodeficiency virus-infected patients. Gastroenterology 2005;128:313-27. [PubMed: 15685543]

34. Sarrazin C, Kieffer TL, Bartels D, et al. Dynamic hepatitis C virus genotypic and phenotypic changes in patients treated with the protease inhibitor telaprevir. Gastroenterology 2007;132:1767-77. [PubMed: 17484874] 
35. Pawlotsky JM, Dahari H, Neumann AU, et al. Antiviral action of ribavirin in chronic hepatitis C. Gastroenterology 2004;126:703-14. [PubMed: 14988824]

36. Zeuzem S, Herrmann E, Lee JH, et al. Viral kinetics in patients with chronic hepatitis $\mathrm{C}$ treated with standard or peginterferon alpha2a. Gastroenterology 2001;120:1438-47. [PubMed: 11313314]

37••. Pilli M, Zerbini A, Penna A, et al. HCV-specific T-cell response in relation to viral kinetics and treatment outcome (DITTO-HCV project). Gastroenterology 2007;133:1132-43. [PubMed: 17919489]This study shows that better baseline HCV specific CD8 cell proliferative responses are correlated with fast second phase viral decay ( $\geq 0.09 /$ day). Moreover, it suggests that IFN/RBV therapy is unable to induce a rapid restoration of an efficient adaptive T-cell response, which supports modeling predictions shown in reference 50.

38. Inoue K, Umehara T, Ruegg UT, et al. Evaluation of a cyclophilin inhibitor in hepatitis C virusinfected chimeric mice in vivo. Hepatology 2007;45:921-8. [PubMed: 17393519]

39. Dahari H, Perelson AS. Hepatitis $\mathrm{C}$ virus kinetics in chimeric mice during antiviral therapy. Hepatology 2007;46:2048-9. [PubMed: 17935224]

40. Lindh M, Alestig E, Arnholm B, et al. Response prediction and treatment tailoring for chronic hepatitis C virus genotype 1 infection. J Clin Microbiol 2007;45:2439-45. [PubMed: 17581934]

41. Dahari H, Perelson AS. Hepatitis C virus RNA kinetics: Drug efficacy and the rate of HCV-infected cells loss. World J Gastroenterol 2007;13:3020-1. [PubMed: 17589958]

42. Levy-Drummer RS, Haagmans BL, Soulier A, et al. Pharmacodynamic modeling of HCV kinetics during peg-interferon-alfa-2a (40KD) and ribavirin treatment of chronic hepattits $\mathrm{C}$ genotype 1 patients in the DITTO-HCV study. Hepatology 2004;40:390A.

43. Bekkering FC, Neumann AU, Brouwer JT, et al. Changes in anti-viral effectiveness of interferon after dose reduction in chronic hepatitis $\mathrm{C}$ patients: a case control study. BMC Gastroenterol 2001;1:14. [PubMed: 11801193]

44. Dahari H, Lo A, Ribeiro RM, Perelson AS. Modeling hepatitis C virus dynamics: Liver regeneration and critical drug efficacy. J Theor Biol 2007;247:371-81. [PubMed: 17451750]

45. Wein LM, D'Amato RM, Perelson AS. Mathematical analysis of antiretroviral therapy aimed at HIV-1 eradication or maintenance of low viral loads. J Theor Biol 1998;192:81-98. [PubMed: 9628841]

46. Callaway DS, Perelson AS. HIV-1 infection and low steady state viral loads. Bull Math Biol 2002;64:29-64. [PubMed: 11868336]

47. Pal S, Shuhart MC, Thomassen L, et al. Intrahepatic hepatitis C virus replication correlates with chronic hepatitis C disease severity in vivo. J Virol 2006;80:2280-90. [PubMed: 16474135]

48. Everson GT, Hoefs JC, Seeff LB, et al. Impact of disease severity on outcome of antiviral therapy for chronic hepatitis C: Lessons from the HALT-C trial. Hepatology 2006;44:1675-84. [PubMed: 17133499]

49. Iacobellis A, Siciliano M, Perri F, et al. Peginterferon alfa- $2 \mathrm{~b}$ and ribavirin in patients with hepatitis C virus and decompensated cirrhosis: a controlled study. J Hepatol 2007;46:206-12. [PubMed: 17125876]

50•. Dahari H, Ribeiro RM, Perelson AS. Triphasic decline of hepatitis C virus RNA during antiviral therapy. Hepatology 2007;46:16-21. [PubMed: 17596864]This theoretical paper explains why some patients exhibit a triphasic viral decay without assuming a delayed immune response due to IFN or RBV. 


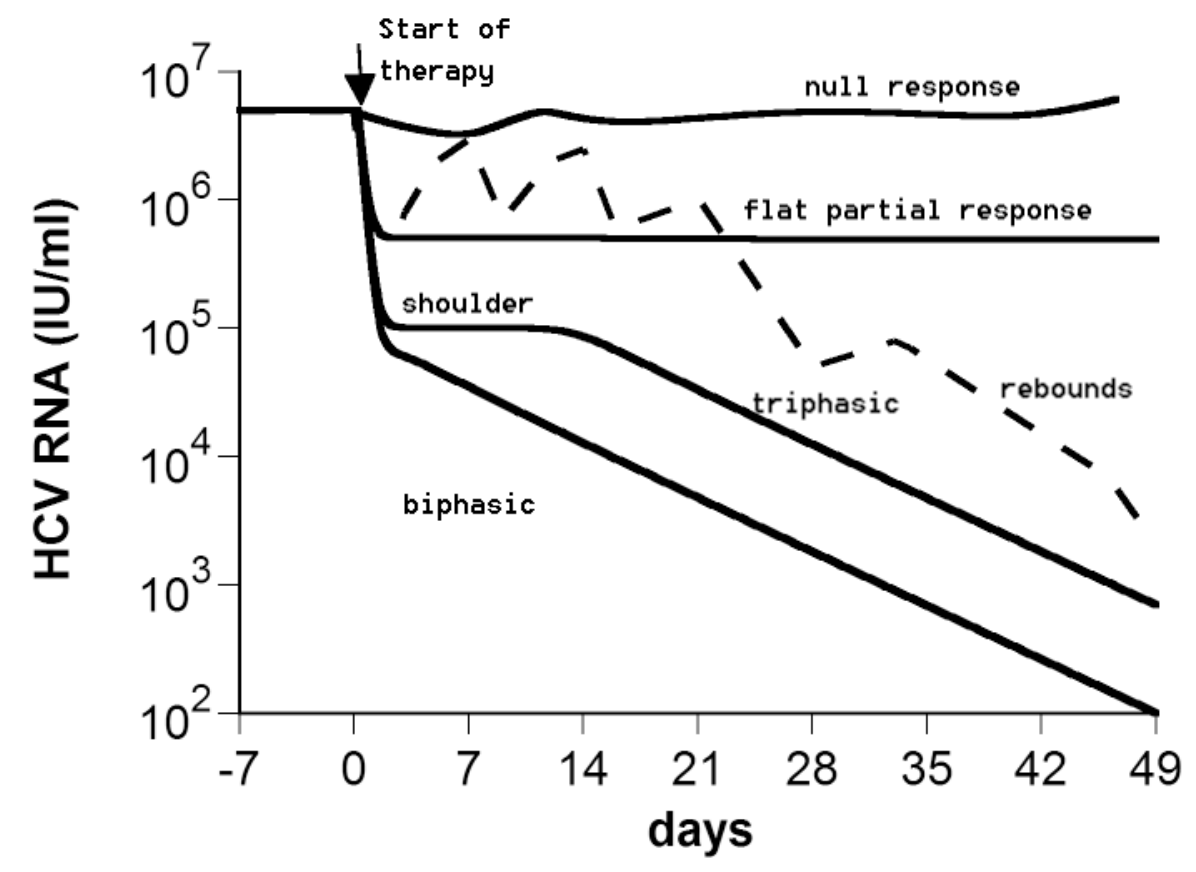

Figure 1.

Examples of different HCV RNA profiles during therapy 


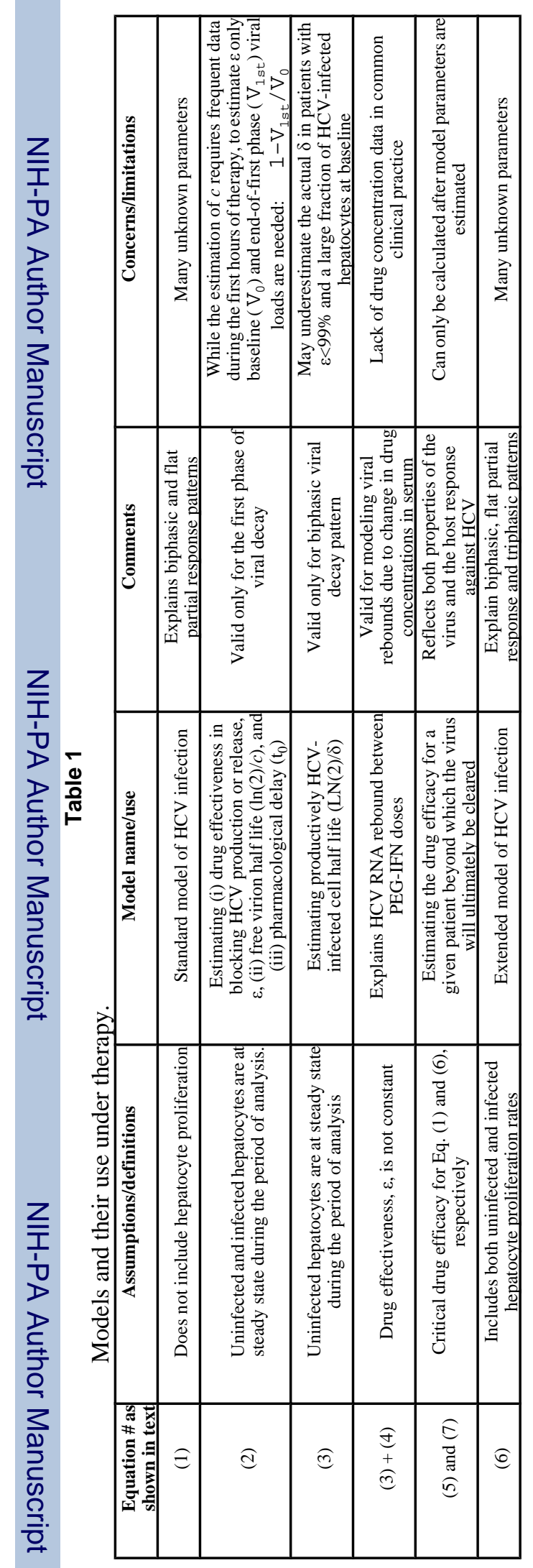

Curr Hepat Rep. Author manuscript; available in PMC 2009 January 14. 


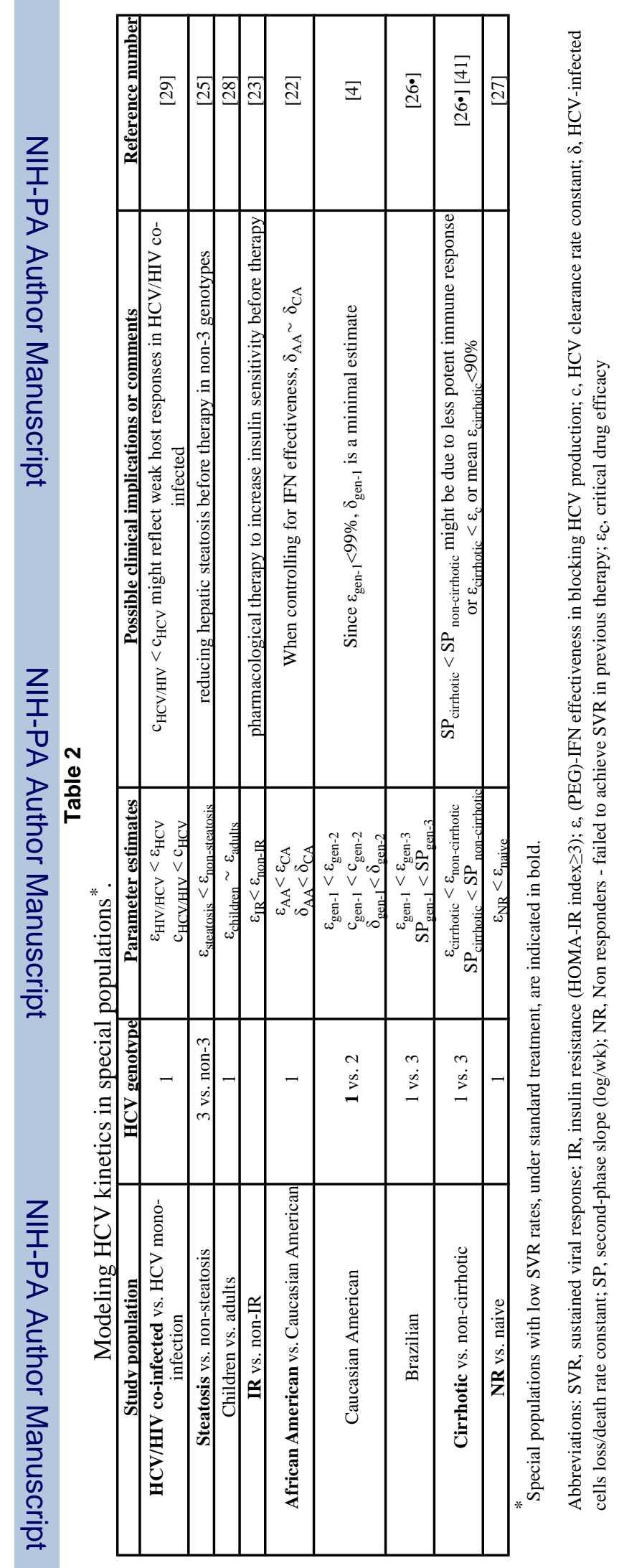

Curr Hepat Rep. Author manuscript; available in PMC 2009 January 14. 